\title{
HIGHLIGHTS
}

IMAGING

\section{Visualizing intraepithelial bacteria in vivo with confocal endomicroscopy}

Investigators have successfully used fluorescein-enhanced confocal laser endomicroscopy (CLE) to observe the in vivo localization of bacteria within the mucosal layers of the colon and ileum.

"There is accumulating evidence that IBD is the result of an abnormal immune response to gut bacteria," explains Alastair Watson, the study's corresponding author. "The development of a technique to visualize bacteria in lightly sedated patients would be a useful diagnostic tool that could be used to investigate this hypothesis further," he notes.

Intramucosal bacteria can also be detected by bacterial culture, electron microscopy and immunohistochemistry; however, these methods are dependent on mucosal biopsy, which is not only time-consuming but also disrupts the mucosal architecture.

The method used in this study relies on the administration of intravenous fluorescein, which leaks out of the mucosal blood vessels and is absorbed by bacteria. The latter can subsequently be visualized in vivo with CLE. Watson and colleagues found that fluorescein-aided CLE could detect intramucosal bacteria in humans with a sensitivity of $89 \%$ and a specificity of $100 \%$ compared with fluorescence in situ hybridization, the gold-standard technique.

The researchers initially analyzed archived fluorescein-enhanced CLE images from 113 patients in remission from IBD (84 patients with ulcerative colitis and 29 with Crohn's disease) and 50 IBD-free controls. Intramucosal bacteria were present in considerably more patients than controls (63\% versus $14 \%$, respectively). They also conducted a prospective study in 10 patients with ulcerative colitis, 10 with Crohn's disease, and 10 IBD-free individuals. The distribution of colonic and ileal

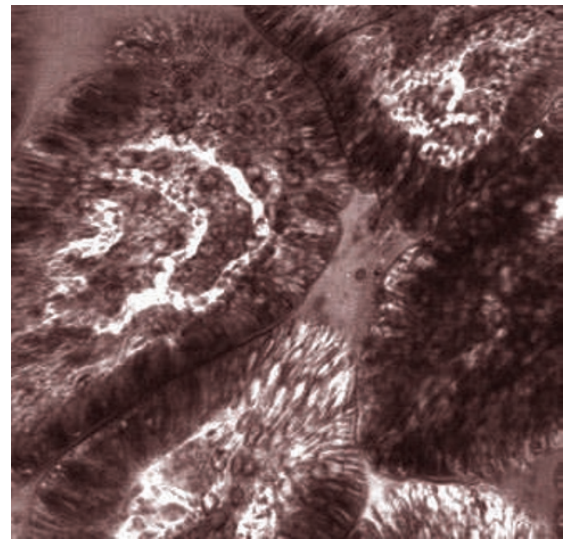

Confocal endomicroscopic image of the small intestine from a patient with Crohn's disease. Courtesy of A. J. Watson.

intramucosal bacteria was considerably more extensive in patients than controls.

Watson's team is developing other diagnostic uses for fluorescein-aided CLE, including a technique for assessing the integrity of intestinal epithelium.

Shreeya Nanda

Original article Moussata, D. et al. Confocal laser endomicroscopy is a new imaging modality for recognition of intramucosal bacteria in inflammatory bowel disease in vivo. Gut 60, 26-33 (2011) 